\title{
Circuit
}

Musiques contemporaines

\section{Portrait de Michel Longtin}

Entretien

\section{Yannick Delbecque}

Volume 14, numéro 2, 2004

Montréal/Nouvelles Musiques

URI : https://id.erudit.org/iderudit/902319ar

DOI : https://doi.org/10.7202/902319ar

Aller au sommaire du numéro

Éditeur(s)

Les Presses de l'Université de Montréal

ISSN

1183-1693 (imprimé)

1488-9692 (numérique)

Découvrir la revue

Citer ce document

Delbecque, Y. (2004). Portrait de Michel Longtin : entretien. Circuit, 14(2),

113-122. https://doi.org/10.7202/902319ar

Ce document est protégé par la loi sur le droit d'auteur. L'utilisation des services d'Érudit (y compris la reproduction) est assujettie à sa politique d'utilisation que vous pouvez consulter en ligne.

https://apropos.erudit.org/fr/usagers/politique-dutilisation/
Cet article est diffusé et préservé par Érudit.

Érudit est un consortium interuniversitaire sans but lucratif composé de l’Université de Montréal, l'Université Laval et l'Université du Québec à Montréal. Il a pour mission la promotion et la valorisation de la recherche. https://www.erudit.org/fr/ 


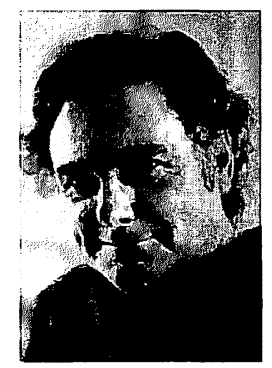

\title{
Portrait de Michel Longtin
}

\section{Entretien}

\author{
Yannick Delbecque
}

C'est à la suite d'un repas champêtre dans la petite ville de Rawdon que l'entretien suivant avec Michel Longtin et quelques-uns de ses amis personnels a eu lieu. La discussion s'est déroulée de manière très informelle, et toutes les personnes présentes y ont participé à un moment ou l'autre. La familiarité et l'esprit de jeu que l'on retrouve ordinairement lors d'une rencontre amicale de cette sorte rendent justice au caractère et aux idées de Michel Longtin, voire même à son approche de la composition et de l'enseignement.

Michel Longtin pratique le pragmatisme musical en se tenant éloigné des débats intellectuels et esthétiques de toutes sortes pour se concentrer sur la création. II aime choquer par ses propos, pour faire réaliser à qui sait lire entre les lignes que se prendre au sérieux est incompatible avec l'émerveillement nécessaire pour découvrir et créer. Cela en fait une bête peu docile à approcher avec un microphone et des questions...

Michel Longtin - C'est sur quoi l'entrevue?

Yannick Delbecque - Ce n'est pas très clair.

$\mathrm{ML}$ - Quaternions?

YD - Pas nécessairement sur Quaternions. Ce qu'on veut, c'est une entrevue sur toi, Michel.

$M L$ - [Rires] Non, non! ॥ n'y en est pas question!

YD - Je me suis dit que je ne peux pas te faire redire ce que tu as dit des milliers de fois et ce que les gens peuvent lire un peu partout.

$M L$ - Je ne suis apparu nulle part! [Rires] Bon, je m'intéresse à Jerry Goldsmith.

Françoise Delbecque - Est-ce que c'est notoire? 
$\mathrm{ML}-$ Ce n'est pas high class...

YD - Te sertil dans ta musique?

$\mathrm{ML}$ - J'ai connu Jerry Goldsmith à une époque où je commençais a peine à écouter de la musique classique, et je trouvais qu'il y avait des sonorités chez ce garslà... Je donne un exemple : il a commencé dans les années 1960, et un des films que j'ai vu en 1967, c'est The Flim-Flam Man. Ça commençait par une "tounne" d'harmonica, il y avait aussi un banjo; après il y avait des cordes symphoniques qui rentraient. Tout un blend de sons... un orchestre, mais pas conventionnel. Un orchestre symphonique avec du banjo et de l'harmonica, et ça marchait mon vieux, ça marchait! II n'y a pas un compositeur de musique contemporaine qui va faire quelque chose comme ça.

Tu pars de ça, tu passes par Papillons, en 1972, où c'est très impressionniste (c'est du Ravel, mais bien sûr avec une touche personnelle), tu t'en vas à Alien, en 1978 (bien sûr, il y a Penderecki là-dedans)... En fin de compte, ta vraie orchestration, ta vraie couleur, c'est là que tu l'as apprise. C'est pas dans les bouquins. Tu écoutes Schumann, tu regardes la partition, ensuite tu écoutes Schubert, tu regardes la partition : c'est bien dur à différencier! C'est écrit à peu près pareil. Mais avec Goldsmith, tu fais du solfège de l'oreille : ce sont ses cors qu'il fait ressortir de tous ses cuivres, une petite note de piccolo à tel endroit donne un effet de métal... $\mathrm{Si}$ j'ai appris à orchestrer, c'est en écoutant Jerry Goldsmith. Je n'ai pas appris à orchestrer avec Stockhausen, avec Boulez, avec les grands noms et les grosses légumes, mais avec un "vulgaire compositeur de musique de film", comme les bons pensants se plaisent à dire.

YD - C'est quelqu'un qui travaille constamment avec ce matériel orchestral...

$\mathrm{ML}$ - \|n'arrête pas une seconde!

YD - ... et qui a eu le temps de digérer à fond l'orchestration traditionnelle, qui est allée à l'essentiel.

$M L$ - C'est qu'il fait à peu près six musiques de 80 minutes par année pour orchestre, alors que [ton faussement snob] les grands compositeurs prennent deux ans et passent à la radio: "J'ai composé une pièce pour orchestre, et la la la... une pièce de 20 minutes... » [voix normale] pis ça pète, pis ça pète, pis ça pète... Goldsmith, il ne passe pas à la radio, il n'a pas le temps!

Marie Robert - Tu as déjà dit que Goldsmith, c'est une chose dont on ne parle pas dans ton cercle, on ne considère pas ces gens-là comme des gens érudits du point de vue musical. Or, c'est tout le contraire d'après toi : quelqu'un comme Goldsmith doit forcément très bien connaître la musique.

$M L$ - Oui, et toutes les musiques. Je connais bien des professeurs d'université qui en savent même pas le dixième de ce Goldsmith sait en musique.

MR - Qu'est-ce qui est la source de l'aveuglement de certaines personnes vis-à-vis de ces musique là? 
$M L$ - C'est le fait qu'ils ont été éduqués à la fine pointe d'une pensée musicale très avancée, très intellectuelle, très savante, et que, en fin de compte, tout ce qui est autre chose que cela ne vaut pas la peine de s'y intéresser.

MR - Cela ne fait pas partie de leur monde.

$M L$ - Cela n'en vaut pas la peine : ça manque d'intellect! Une musique doit être structurée de façon intelligente, et tout ça... Or, on a bien vu depuis les années 1950 que ce n'est pas la structure d'une œuvre qui fait qu'elle passe la rampe, qu'elle est de la bonne musique. II y a un insondable dans la musique. C'est un blend " de toutte » : la structure, les idées musicales de départ, l'inspiration... Serge Garant, je me souviens très bien, à l'époque où il enseignait, il ne fallait pas dire le mot "inspiration", parce que cela n'existait plus l'inspiration. II ne fallait pas le dire parce que ça appartenait à l'ancienne musique, la musique romantique. II ne fallait pas que tu aies de l'inspiration, il fallait plutôt que tu cherches ta structure et que tu la trouves, et enfin tu pouvais composer. Je regrette, mais tout ça, c'est de l'inspiration... Changeons de mots : inspiration, intuition... tout ça, c'est la même affaire!

YD - Michel, un peu en rapport avec tout cela, quand tu critiques tes propres pièces et que tu dis : "Cette pièce-là, je l'ai ratée", sur quoi te bases-tu pour dire ça?

$\mathrm{ML}$ - L'oreille.

YD - Oui, mais que trouves-tu qui ne va pas? Tu trouves qu'elle n'exprime pas ce que tu voulais, tu trouves qu'elle n'est pas équilibrée comme tu voulais?

ML - Elle n'exprime pas ce que je voulais, d'une part. Deuxièmement, elle ne marche pas comme j'aurais souhaité, elle ne fonctionne pas comme je pensais qu'au départ qu'elle fonctionnerait. Pourquoi est-ce arrivé dans plusieurs de mes pièces? En vieillissant, je me suis aperçu que souvent je composais des pièces fatigué, pressé de les finir, stressé en les faisant, et cela ne donne pas le même résultat qu'une pièce composée calmement, en prenant ton temps pour bien goûter ce que tu veux exprimer. Michel Garneau, un des grands écrivains de théâtre, disait : "Il y a une pièce que je vais enlever de mon répertoire, je l'ai stressée celle-là». Ça veut tout dire. Tu sais, tu fais une sculpture en bois, et à un moment donné, boom! La moitié du bout de bois sacre son camp! Tu l'as stressé celuilà.

Luc Godin - Quand tu as entendu Quaternions, as-tu trouvé ça bon?

$\mathrm{ML}$ - J'étais content du résultat, et je vais te dire pourquoi : je m'attendais à ce que ce soit une catastrophe. Disons qu'ils ont été honnêtes. Ils ont donné une bonne idée de ce que ça pouvait être. C'est pas ce que ç'aurait été si tu avais eu l'appareillage de Deutsche Grammophon, un chef d'orchestre et la grosse machine en arrière, et le temps. Étant donné les circonstances, c'était honnête. 
$\mathrm{ML}$ - Les mathématiques.

YD - Les mathématiques?

$\mathrm{ML}-J^{\prime}$ utilise tout le temps des mathématiques, tout le temps. C'est absolument faux! Souvent j'utilise une entité mathématique, une équation, une "affaire" que je "bizounne» avec le peu que je connais... Par exemple, dans Hommage à Euler, i'ai fait la structure des 23 tableaux (calculé la durée de chacun), avec des équations que j'ai fabriquées à partir de Mandelbrot, j'ai mêlé ça avec l'équation de Robert May, j'ai fait une toile d'araignée en puissance quatre et cela a donné des résultats assez l'fun merci. J'ai utilisé des mathématiques là-dedans, mais autrement, à part les pièces pour percussion où j'ai utilisé la stochastique, parce que les idées avaient besoin de cet outillà, quand j'utilise les mathématiques, c'est simplement pour me donner le temps de faire venir des idées musicales. Une fois que les idées musicales sont dessinées dans ton petit livre, tu commences à faire ta structure avec supposément des mathématiques, tu vois que ça marche pas, alors t'envoies tout ça chez l'diable, pis là, tu commences à composer !

YD - C'est sûrement un peu comme ça que l'on peut expliquer comment tu peux réconcilier tes influences musicales avec des choses plus abstraites comme les mathématiques.

$M L$ - Oui. J'aime les mathématiques parce que je trouve que les mathématiques, c'est un des arts suprêmes. C'est un Art, les mathématiques, c'est du Grand Art. Bon, [ton faussement snob] plutôt que d'avoir la barbe longue et de puer, les mathématiciens portent la cravate.

YD - [Rires] II faudra que je t'en présente quelques autres...

$\mathrm{ML}$ - [Voix normale] Enfin les mathématiciens assument leur identité d'artiste! Ils assument qu'il y a quelque chose là qui vient de l'intuition! [Rires]

YD - $\|$ y a toujours une différence : on est libéré du passé en mathématiques. On n'a pas constamment à se demander, parce qu'un tel a fait ça dans tel style, ou écrit sa preuve de telle façon, si on doit faire pareil ou pas. C'est un poids de moins sur les épaules.

$M L$ - Toi, par exemple, si tu es aux prises avec un problème, et que tu as besoin d'un outil qui vient de 1772, d'un autre outil qui vient de 1926 et d'un autre qui vient de 1968, tu te feras pas de complexes pour ça.

YD - Pas une seule seconde.

$M L$ - Tu vas faire ça et arriver à tes fins, et voilà ! On n'est pas encore arrivé là en musique ni en peinture ni en art en général.

YD - Cela ressemble à quoi, une idée de départ pour toi ? Ça se manifeste comment?

$M L$ - Tu l'entends. 
YD - Ce sont des bribes mélodiques, des couleurs, des bouts d'orchestration, des transitions?

$M L$ - Selon l'idée, ça peut être justement des couleurs timbrales, ça peut être une idée harmonique, une idée d'une mélodie, avec un accompagnement spécifique, qui va être développée plus loin. Comme i'ai fait 10 ans d'électroacoustique... Tu sais, quand tu as trois heures de studio par semaine, c'est pas en entrant dans le studio, avec les milliers d'appareils que tu as là (qu'il faut que tu "plogues", ce qui prend déjà trois heures) qu'il faut que tu te demandes ce que tu vas composer, parce que tu n'y arriveras jamais. Tu fais des sons, tu cherches des sons, et à un moment donné tu as une banque de sons que tu trouves intéressants, avec lesquels tu pourrais travailler pour faire un certain genre de "tounne". Pour que tu te rappelles que sur la piste numéro un il y a tel genre de son, tu lui fais un petit graphique. Un deuxième son, tu lui fais un autre graphique, un troisième son très en dents de scie, alors tu lui fais un graphique en dents de scie... Tu penses à ta pièce et éventuellement tu te dis : elle va commencer avec ça, avec ce son-là : [Son impossible à transcrire...]. Tu dessines ce que tu entends.

Quand j'ai eu mes idées de musique instrumentale, c'est ce que j'ai fait. J'ai dessiné mes idées. Si j'ai une petite idée, disons une page dessinée, qui n'est pas développée, je peux très bien le lendemain, que ça soit n'importe où, au café, dans l'autobus, ou... [voix faussement snob] au salon de thé [voix normale], prendre cette idée-là et entendre et prévoir comment ça se continuerait, et de le dessiner!

Une fois que cela est fait, tu fais un deuxième brouillon pour la rythmique, c'està-dire de tout arranger ça dans l'univers de la rythmique et des timbres. C'est un deuxième brouillon, mais plus clean et plus clair. La troisième étape, c'est d'ajouter les hauteurs. Si tu as une idée uniquement harmonique, tu laisses tomber le rythme: tu choisis tes harmonies, et ensuite vient le rythme qui est d'importance mineure.

C'est à cause de l'électronique que j'en suis arrivé à pouvoir composer n'importe où avec mes calepins. Si j'avais attendu d'être chez moi, assis à ma table de travail, et tenté de faire comme Honegger, composer de neuf heures à midi chaque matin, je ne suis pas sûr que chaque matin j'aurais eu les idées qui me sont venues dans l'autobus, au salon de thé ou dans le métro.

YD - Les mathématiques et toutes les autres structures que tu aimes, à quel moment ça entre dans tout ça?

$M L$ - Ordinairement, ça entre au début.

YD - À l'étape du dessin, de la composition?

$M L$ - Avant le dessin, parce qu'il y a certaines équations mathématiques qui tout à coup m'enchantent. Pourquoi? Je ne le sais pas. Parce que leurs créateurs, je les trouve bien intelligents, parce que je réussis à me faire "accroire " que telle équation a un rapport avec la pièce que je veux composer sur [ton ironiquement langoureux] 
l'amour. [Voix normale] Éventuellement, quand j'ai terminé de me faire mon cinéma, les idées musicales commencent à apparaître. Je suis convaincu que ce que j'ai fait auparavant avec les maths influence d'une manière ou l'autre mes idées musicales.

YD - Tu utilises tout de même les mathématiques à d'autres étapes. Tu m'as déjà dit par exemple avoir déterminé les longueurs des sections dans Quaternions par un jeu avec les quaternions.

$M L$ - Oui.

YD - Ça intervient donc à d'autres niveaux.

$M L$ - Regarde par exemple Autour d'Ainola pour orchestre, c'a été fait avec quelque chose de fort primaire. Vu que c'est un hommage à Sibelius, j'ai pris les sept symphonies de Sibelius par Bernstein. Première symphonie, les mouvements durent tant, deuxième symphonie durent tant, troisième symphonie durent tant... J'ai noté ça pour les sept symphonies, et à chaque début de mouvement, je tirais une ligne verticale. C'étaient les durées des mouvements des symphonies qui déterminaient combien d'étapes il y allait y avoir dans la pièce et combien de temps j'allais consacrer à chaque étape. Jusqu'à un certain point, c'est comme le yin et le yang : même si tu as déterminé la forme de ta pièce sur le plan de la longueur, ces longueurs là te tracent le chemin pour te dire que la prochaine idée, ça doit être une idée courte, et s'il y a une longue idée qui s'en vient, peut-être que ça serait le temps de mettre un silence. Donc ces petits jeux-là t'aident aussi à trouver tes idées musicales.

FD - Ca t'évite de chercher parce que ça te fait un petit guide...

ML - C'est très bien... Ça m'évite de chercher complètement. [Rires]

FD - Est-tu obligé de t'en tenir à ça?

$\mathrm{ML}$ - Pas obligé du tout!

YD - Je sais que tu te sers de structure comme celle-là au point de vue rythmique parfois, pour générer des séquences rythmiques que tu pourrais utiliser.

$\mathrm{ML}$ - Dans la dernière pièce, Attracteurs vers Xenakis...

FD - [Rires] J'ai entendu : "En tracteur avec Xenakis!»

$M L$ - C'est à peu près ça que ç'a donné comme sonorité, si tu veux savoir... [Rires] $C^{\prime}$ est pas une pièce que j'ai bien réussie. Le NEM l'a bien jouée, mais moi j'ai pas réussi cette pièce-là.

MR - Tu allais dire quelque chose sur la rythmique, en utilisant Xenakis comme exemple.

$M L$ - Avec Xenakis, tu ne sais jamais ce qui va arriver d'une seconde à l'autre, alors que chez certains compositeurs, d'après leur style, on peut se mettre dans un état d'écoute où on connaît le genre de surprise qu'il va faire, ou le genre de sonorité qu'il va y avoir. Mais avec Xenakis, on ne sait jamais d'un instant à l'autre ce qu'il 
peut arriver; même si parfois ce sont des nuages sonores plus prévisibles, il peut toujours y avoir une surprise très spéciale. C'est dans cet espritlà que, par exemple, je me suis fait des mélodies à l'aide de la distribution de Cauchy où il y a un paquet de petites notes rapides et une tenue assez longue : "pétipatatooo »... [ML donne ici un exemple sonore impossible à transcrire...]

YD - Ça va être bon dans Circuit, ça! [Rires]

$\mathrm{ML}-\mathrm{C}^{\prime}$ est pour faire des surprises mélodiques. Comme tu sais, Cauchy c'est $0,0,1,0,0,1,1,1,0,0,1,1,1,0,46 \ldots$ J'ai toujours été fasciné par cette distributionlà, parce qu'elle engendre des surprises... alors que la Gaussienne, il n'y a pas grand-surprises à avoir : tout est clean.

YD - Quand tu arrives à l'étape de choisir tes hauteurs, si tu n'as pas trop de contraintes harmoniques ou autres du même genre, comment procèdes-tu?

$M L$ - Ça dépend des mouvements. Si, par exemple, tu prends le mouvement «Émilie » dans Quaternions, comme je parle d'Émilie qui va mourir et tout ça, je vais carrément faire une mélodie comme, j'imagine, Brahms, Wagner ou n'importe qui s'y prenait pour composer une mélodie. Je vais essayer de la chercher, de lui trouver une caractéristique, de la développer... C'est mélodique, donc tu la fais comme les compositeurs faisaient leurs mélodies. Mais si c'est atonal, disons que c'est "Hamilton " ou "Gheorghiu», pour violoncelle solo, tu peux par exemple prendre des modes connus, qui se reproduisent à l'octave, et les défaire. Je reproduis à partir de la $7^{\mathrm{e}}$ mineure certains modes, ainsi de suite. Ça m'élabore donc une série de notes dans laquelle je peux piger, mais à chaque octave il en manque. De là, je peux faire des contours qui ont une sonorité différente d'une mélodie normale, qui sont peutêtre axés sur la quarte plutôt que sur le diatonisme. Ça dépend du sujet.

YD - Je me demandais si, parfois, la musique de film ne t'aidait pas un peu à trouver une mélodie, un air qui exprime ce que tu veux.

$\mathrm{ML}$ - Non. Ce n'est pas à ce niveaulà que la musique de film va m'aider.

YD - C'est plus pour la couleur?

ML - C'est sur le plan de l'orchestration. Carrément. Une mélodie, quand tu veux en faire une, si tu veux qu'elle ressemble à du Schumann, tu vas aller voir Schumann, voir ses caractéristiques, mais ordinairement, quand je fais mes «tounnes" mélodiques tonales, je ne veux pas qu'elle ressemble à personne. Je veux qu'elle ressemble à ce que j'entends dans ma tête, bien qu'on ne soit pas à l'abri des influences.

YD - Je voudrais parler du théâtre. Quand on lit des choses sur toi, on mentionne cela souvent, mais j'aimerais savoir quel rôle le théâtre et le mime jouent dans ce que tu fais maintenant, dans ta musique. Est-ce qu'il y a des idées musicales qui te viennent de là, ou une manière de penser, d'agencer tes idées? 
$M L$ - Non. Je n'étais pas assez mature quand j'en ai fait pour que ça m'influence, je n'en ai pas fait assez longtemps pour que ça m'influence. J'ai toujours le réflexe de regarder le jeu des acteurs quand je décide de voir un film. Pour moi, c'est très important. II se peut que j'aille voir un film, pas pour l'action, pas pour l'histoire, mais pour le comédien, pour voir comment il va s'en sortir dans le rôle qu'on lui a donné.

Je ne sais pas pourquoi, mais on dirait que ça m'a suivi. Depuis le moment où j'ai fait du mime, on dirait qu'il y a une nostalgie de cette discipline-là, de cet artlà - j'avais toujours voulu en faire - et ma façon de pallier ça est de voir comment certains grands comédiens vont être créateurs en inventant leurs personnages.

MR - [À tous] Si Michel était un mime qui avait potentiellement beaucoup de talent, il y a aussi tout le côté de son caractère qui aime rire. Il est excellent imitateur par exemple. [À ML] Est-ce que ça se transmet dans ta musique? J'ai remarqué que souvent dans ta musique, par exemple dans Hommage à Euler, il y a tous ces côtés très bouffons.

ML - Mais là, ça fait partie du concept de 007!

MR - Tu penses pas qu'il y en ait à d'autres moments aussi ?

ML - Non, parce que si c'était ça, j'aurais fait comme Michel Smith, c'est-à-dire que j'aurais fait une musique théâtrale, où il y a une action théâtrale qui se passe, soit conventionnelle, soit complètement " pétée», surréaliste, qui te fait bidonner en même temps qu'il y a de la musique. Là, on pourrait dire que ce qui m'est arrivé quand j'étais plus jeune et que je faisais du théâtre m'a poursuivi. Ce n'est vraiment pas ça qui est arrivé. Ca ne m'a pas poursuivi dans ma musique, sauf que dans ma musique, je raconte toujours des histoires [rires], ce que les autres ne font pas. Ca a fait dire à Robert Léonard, il y a 18 ans de ça, que j'étais la petite tante Lucille de la musique contemporaine au Québec [rires]. C'était pas un compliment. Je l'ai pris en riant; i'ai trouvé ça très drôle. Je ne m'attendais pas à ça.

MR - Tu te racontes toujours des histoires?

$\mathrm{ML}$ - Je me raconte des histoires. J'y crois l'espace d'une nanoseconde et quand je retourne à la réalité, c'est moins drôle.

YD - Donc, ta musique n'est pas théâtrale, pas narrative, mais elle raconte des histoires?

$\mathrm{ML}$ - Des fois, elle est narrative!

YD - Oui, il y en a pour lesquelles c'est plus explicite que d'autres. Pour Quaternions, il y a même un texte associé à la musique. Astu d'autres exemples pour lesquels tu penses que c'est narratif?

ML - Mon doux, mon Dieu! Je pense....

MR - Conrad est narrative. 
YD - Pour la La route des pèlerins reclus, tu t'es inspiré d'un texte, mais est-ce que tu y racontes une histoire?

$M L$ - Je fais des tableaux. Je prends six extraits de l'histoire et j'en fais une espèce de... pour bien parler, une "suite symphonique". Alors tu comprends, je suis une espèce de romantique attardé. Je suis né 75 ans trop tard, mais qui [ton faussement snob] selon certains musicologues bien cotés [voix normale] est en avance de 20 ans sur son temps. Ou bien je suis 75 ans en retard, ou bien 20 ans en avance... [Ton faussement snob] Choisissez selon vos esthétiques...

MR - Quel est le rôle du vélo dans ta composition?

$\mathrm{ML}$ - [Voix normale] De me tenir en santé pour «toffer la run» le plus longtemps possible.

LG - Comment tu t'y prends pour monter une côte?

$M L$ - Je me mind. Je me dis: "t'as pas mal, t'as pas mal», bien que les jambes veulent me péter en douze. Pis d'un coup [voix essoufflée], tu deviens essoufflé. [Voix normale] Pas de problèmes! II y en a de l'oxygène. Pas de problèmes! Un moment donné le "bicycle" veut arrêter, parce les jambes ne sont plus capables. Elle est finie la côte! Je finis par la monter, mais pendant que je fais tout ça, y en a qui la monte : ziiiiif! C'est comme si je faisais rien!

Pour composer Attracteur vers Xenakis, j'ai vu les trois faces quand je montais une côte : Xenakis, Trenet, et mon père. Mon père sacrait : "Vas-tu la monter la "tabarnak" de côte! » C'est terrible. Plus tu vieillis, moins tu es supposé monter de côtes.

LG - À Rawdon, il n'y a rien que des côtes! 

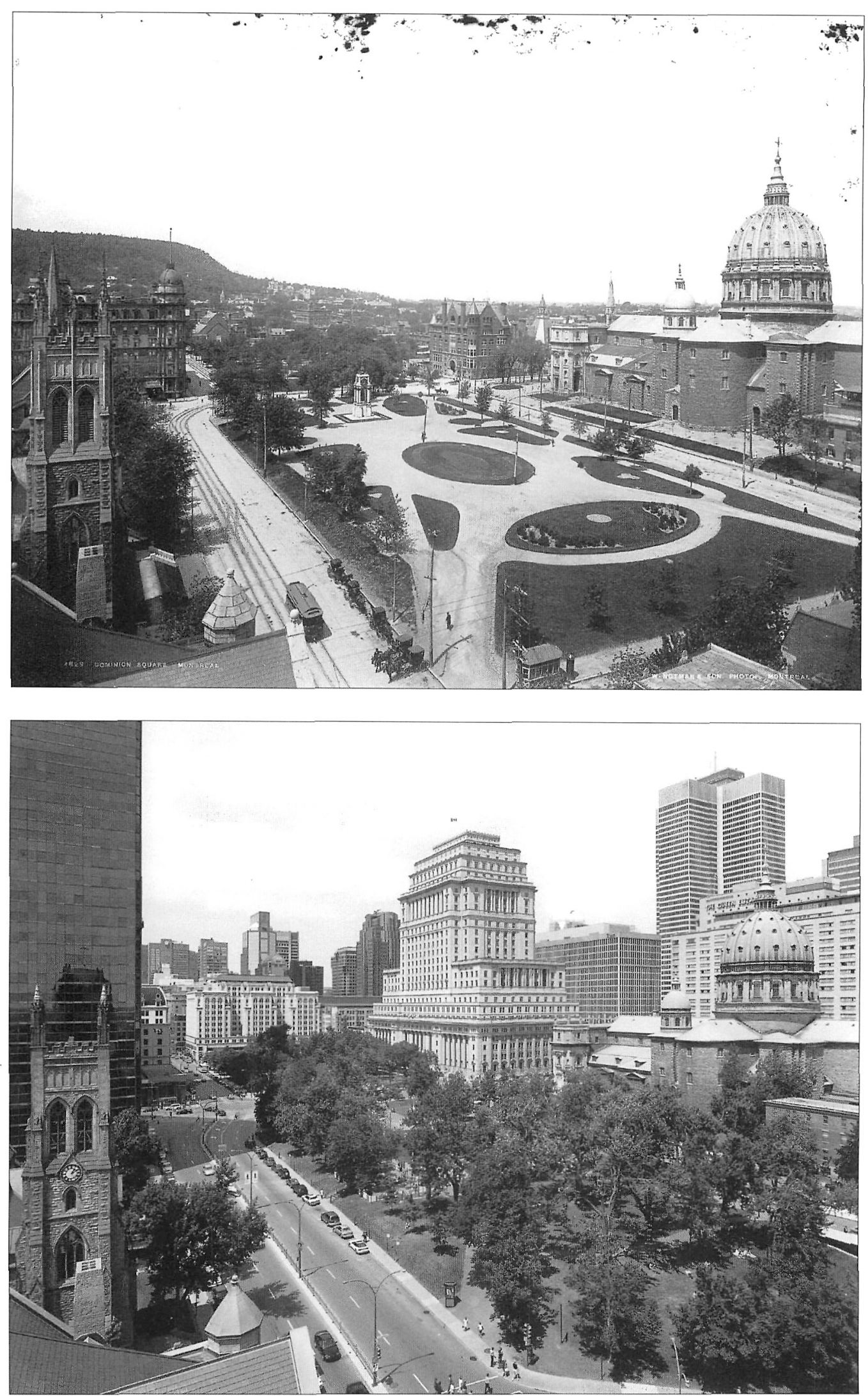